\title{
Computing longest common substring and all palindromes from compressed strings
}

\author{
Wataru Matsubara ${ }^{1}$, Shunsuke Inenaga ${ }^{2}$, Akira Ishino $^{1}$, Ayumi Shinohara $^{1}$, \\ Tomoyuki Nakamura $^{1}$, and Kazuo Hashimoto ${ }^{1}$ \\ ${ }^{1}$ Graduate School of Information Science, Tohoku University, Japan \\ \{matsubara@shino., ishino@, ayumi@, nakamura@aiet., \\ hk@aiet.\}ecei.tohoku.ac.jp \\ 2 Department of Computer Science and Communication Engineering, \\ Kyushu University, Japan \\ inenaga@c.csce.kyushu-u.ac.jp
}

\begin{abstract}
This paper studies two problems on compressed strings described in terms of straight line programs (SLPs). One is to compute the length of the longest common substring of two given SLP-compressed strings, and the other is to compute all palindromes of a given SLPcompressed string. In order to solve these problems efficiently (in polynomial time w.r.t. the compressed size) decompression is never feasible, since the decompressed size can be exponentially large. We develop combinatorial algorithms that solve these problems in $O\left(n^{4} \log n\right)$ time with $O\left(n^{3}\right)$ space, and in $O\left(n^{4}\right)$ time with $O\left(n^{2}\right)$ space, respectively, where $n$ is the size of the input SLP-compressed strings.
\end{abstract}

\section{Introduction}

The importance of algorithms for compressed texts has recently been arising due to the massive increase of data that are treated in compressed form. Of various text compression schemes introduced so far, straight line program (SLP) is one of the most powerful and general compression schemes. An SLP is a context-free grammar of either of the forms $X \rightarrow Y Z$ or $X \rightarrow a$, where $a$ is a constant. SLP allows exponential compression, i.e., the original (uncompressed) string length $N$ can be exponentially large w.r.t. the corresponding SLP size $n$. In addition, resulting encoding of most grammar- and dictionary-based text compression methods such as LZ-family [1,2], run-length encoding, multi-level pattern matching code [3], Sequitur [4] and so on, can quickly be transformed into SLPs [5-7]. Therefore, it is of great interest to analyze what kind of problems on SLP-compressed strings can be solved in polynomial time w.r.t. $n$. Moreover, for those that are polynomial solvable, it is of great importance to design efficient algorithms. In so doing, one has to notice that decompression is never feasible, since it can require exponential time and space w.r.t. $n$.

The first polynomial time algorithm for SLP-compressed strings was given by Plandowski [8], which tests the equality of two SLP-compressed strings in $O\left(n^{4}\right)$ time. Later on Karpinski et al. [9] presented an $O\left(n^{4} \log n\right)$-time algorithm for 
the substring pattern matching problem for two SLP-compressed strings. Then it was improved to $O\left(n^{4}\right)$ time by Miyazaki et al. [10] and recently to $O\left(n^{3}\right)$ time by Lifshits [11]. The problem of computing the minimum period of a given SLP-compressed string was shown to be solvable in $O\left(n^{4} \log n\right)$ time [9], and lately in $O\left(n^{3} \log N\right)$ time [11]. Gạsieniec et al. [5] claimed that all squares of a given SLP-compressed string can be computed in $O\left(n^{6} \log ^{5} N\right)$ time.

On the other hand, there are some hardness results on SLP-compressed string processing. Lifshits and Lohrey [12] showed that the subsequence pattern matching problem for SLP-compressed strings is NP-hard, and that computing the length of the longest common subsequence of two SLP-compressed strings is also NP-hard. Lifshits [11] showed that computing the Hamming distance between two SLP-compressed strings is \#P-complete.

In this paper we tackle the following two problems: one is to compute the length of the longest common substring of two SLP-compressed strings, and the other is to find all maximal palindromes of an SLP-compressed string. The first problem is listed as an open problem in [11]. This paper closes the problem giving an algorithm that runs in $O\left(n^{4} \log n\right)$ time with $O\left(n^{3}\right)$ space. For second the problem of computing all maximal palindromes, we give an algorithm that runs in $O\left(n^{4}\right)$ time with $O\left(n^{2}\right)$ space.

Comparison to previous work. Composition system is a generalization of SLP which also allows "truncations" for the production rules. Namely, a rule of composition systems is of one of the following forms: $X \rightarrow Y^{[i]} Z_{[j]}, X \rightarrow Y Z$, or $X \rightarrow a$, where $Y^{[i]}$ and $Z_{[j]}$ denote the prefix of length $i$ of $Y$ and the suffix of length $j$ of $Z$, respectively. Ga̧sieniec et al. [5] presented an algorithm that computes all maximal palindromes from a given composition system in $O\left(n \log ^{2} N \times E q(n)\right)$ time, where $E q(n)$ denotes the time needed for the equality test of composition systems. Since $E q(n)=O\left(n^{4} \log ^{2} N\right)$ in [5], the overall time cost is $O\left(n^{5} \log ^{4} N\right)$.

Limited to SLPs, $E q(n)=O\left(n^{3}\right)$ due to the recent work by Lifshits [11]. Still, computing all maximal palindromes takes $O\left(n^{4} \log ^{2} N\right)$ time in total, and therefore our solution with $O\left(n^{4}\right)$ time is faster than the previous known ones (recall that $N=O\left(2^{n}\right)$ ). The space requirement of the algorithm by Gasieniec et al. [5] is unclear. However, since the equality test algorithm of [11] takes $O\left(n^{2}\right)$ space, the above-mentioned $O\left(n^{4} \log ^{2} N\right)$-time solution takes at least as much space as ours.

\section{Preliminaries}

For any set $U$ of pairs of integers, we denote $U \oplus k=\{(i+k, j+k) \mid(i, j) \in U\}$. We denote by $\langle a, d, t\rangle$ the arithmetic progression with the minimal element $a$, the common difference $d$ and the number of elements $t$, that is, $\langle a, d, t\rangle=\{a+$ $(i-1) d \mid 1 \leq i \leq t\}$. When $t=0$, let $\langle a, d, t\rangle=\emptyset$.

Let $\Sigma$ be a finite alphabet. An element of $\Sigma^{*}$ is called a string. The length of a string $T$ is denoted by $|T|$. The empty string $\varepsilon$ is a string of length 0 , namely, $|\varepsilon|=0$. For a string $T=X Y Z, X, Y$ and $Z$ are called a prefix, substring, and 
suffix of $T$, respectively. The $i$-th character of a string $T$ is denoted by $T[i]$ for $1 \leq i \leq|T|$, and the substring of a string $T$ that begins at position $i$ and ends at position $j$ is denoted by $T[i: j]$ for $1 \leq i \leq j \leq|T|$. For any string $T$, let $T^{R}$ denote the reversed string of $T$, namely, $T^{R}=T[|T|] \cdots T[2] T[1]$.

For any two strings $T, S$, let $\operatorname{LCPref}(T, S), \operatorname{LCStr}(T, S)$, and $\operatorname{LCSuf}(T, S)$ denote the length of the longest common prefix, substring and suffix of $T$ and $S$, respectively.

A period of a string $T$ is an integer $p(1 \leq p \leq|T|)$ such that $T[i]=T[i+p]$ for any $i=1,2, \ldots,|T|-p$.

A non-empty string $T$ such that $T=T^{R}$ is said to be a palindrome. When $|T|$ is even, then $T$ is said to be an even palindrome, that is, $T=S S^{R}$ for some $S \in \Sigma^{+}$. Similarly, when $|T|$ is odd, then $T$ is said to be an odd palindrome, that is, $T=S c S^{R}$ for some $S \in \Sigma^{*}$ and $c \in \Sigma$. For any string $T$ and its substring $T[i: j]$ such that $T[i: j]=T[i: j]^{R}, T[i: j]$ is said to be the maximal palindrome w.r.t. the center $\left\lfloor\frac{i+j}{2}\right\rfloor$, if either $T[i-1] \neq T[j+1], i=1$, or $j=|T|$. In particular, $T[1: j]$ is said to be a prefix palindrome of $T$, and $T[i:|T|]$ is said to be a suffix palindrome of $T$.

In this paper, we treat strings described in terms of straight line programs $(S L P s)$. A straight line program $\mathcal{T}$ is a sequence of assignments such that

$$
X_{1}=\operatorname{expr}_{1}, X_{2}=\operatorname{expr}_{2}, \ldots, X_{n}=\operatorname{expr}_{n},
$$

where each $X_{i}$ is a variable and each expr $r_{i}$ is an expression in either of the following form:

$$
\begin{aligned}
& -\operatorname{expr}_{i}=a \quad(a \in \Sigma), \quad \text { or } \\
& -\operatorname{expr}_{i}=X_{\ell} X_{r} \quad(\ell, r<i) .
\end{aligned}
$$

Denote by $T$ the string derived from the last variable $X_{n}$ of the program $\mathcal{T}$. The size of the program $\mathcal{T}$ is the number $n$ of assignments in $\mathcal{T}$.

When it is not confusing, we identify a variable $X_{i}$ with the string derived from $X_{i}$. Then, $\left|X_{i}\right|$ denotes the length of the string derived from $X_{i}$.

For any variable $X_{i}$ of $\mathcal{T}$ with $1 \leq i \leq n$, we define $X_{i}^{R}$ as follows:

$$
X_{i}^{R}= \begin{cases}a & \text { if } X_{i}=a \quad(a \in \Sigma), \\ X_{r}^{R} X_{\ell}^{R} & \text { if } X_{i}=X_{\ell} X_{r} \quad(\ell, r<i) .\end{cases}
$$

Let $\mathcal{T}^{R}$ be the SLP consisting of variables $X_{i}^{R}$ for $1 \leq i \leq n$.

Lemma 1. SLP $\mathcal{T}^{R}$ derives string $T^{R}$.

Proof. By induction on the variables $X_{i}^{R}$. Let $\Sigma_{T}$ be the set of characters appearing in $T$. For any $1 \leq i \leq\left|\Sigma_{T}\right|$, we have $X_{i}=a$ for some $a \in \Sigma_{T}$, thus $X_{i}^{R}=a$ and $a=a^{R}$. Let $T_{i}$ denote the string derived from $X_{i}$. For the induction hypothesis, assume that $X_{j}^{R}$ derives $T_{j}^{R}$ for any $1 \leq j \leq i$. Now consider variable $X_{i+1}=X_{\ell} X_{r}$. Note $T_{i+1}=T_{\ell} T_{r}$, which implies $T_{i+1}^{R}=T_{r}^{R} T_{\ell}^{R}$. By definition, we have $X_{i+1}^{R}=X_{r}^{R} X_{\ell}^{R}$. Since $\ell, r<i+1$, by the induction hypothesis $X_{i+1}^{R}$ derives $T_{r}^{R} T_{\ell}^{R}=T_{i+1}^{R}$. Thus, $\mathcal{T}^{R}=X_{n}^{R}$ derives $T_{n}^{R}=T^{R}$.

Note that $\mathcal{T}^{R}$ can be easily computed from $\mathcal{T}$ in $O(n)$ time. 


\section{Computing Longest Common Substring of Two SLP Compressed Strings}

Let $\mathcal{T}$ and $\mathcal{S}$ be the SLPs of sizes $n$ and $m$, which describe strings $T$ and $S$, respectively. Without loss of generality we assume that $n \geq m$.

In this section we tackle the following problem:

Problem 1. Given two SLPs $\mathcal{T}$ and $\mathcal{S}$, compute $\operatorname{LCStr}(T, S)$.

In what follows we present an algorithm that solves Problem 1 in $O\left(n^{4} \log n\right)$ time and $O\left(n^{3}\right)$ space. Let $X_{i}$ and $Y_{j}$ denote any variable of $\mathcal{T}$ and $\mathcal{S}$ for $1 \leq i \leq n$ and $1 \leq j \leq m$.

\subsection{Overlaps between Two Strings}

For any two strings $X$ and $Y$, we define the set $O L(X, Y)$ as follows:

$$
O L(X, Y)=\{k>0 \mid X[|X|-k+1:|X|]=Y[1: k]\}
$$

Namely, $O L(X, Y)$ is the set of lengths of overlaps of suffixes of $X$ and prefixes of $Y$. Karpinski et al. [9] gave the following results for computation of $O L$ for strings described by SLPs.

Lemma 2 ([9]). For any variables $X_{i}$ and $X_{j}$ of an $S L P \mathcal{T}, O L\left(X_{i}, X_{j}\right)$ can be represented by $O(n)$ arithmetic progressions.

Theorem 1 ([9]). For any $S L P \mathcal{T}, O L\left(X_{i}, X_{j}\right)$ can be computed in total of $O\left(n^{4} \log n\right)$ time and $O\left(n^{3}\right)$ space for any $1 \leq i \leq n$ and $1 \leq j \leq n$.

As we will show in the sequel, we need to compute $O L\left(X_{i}, Y_{j}\right)$ and $O L\left(Y_{j}, X_{i}\right)$ for any $1 \leq i \leq n$ and $1 \leq j \leq m$. In so doing, we produce a new variable $V=X_{n} Y_{m}$, that is, $V$ is a concatenation of SLPs $\mathcal{T}$ and $\mathcal{S}$. Then we compute $O L$ for each pair of variables in the new SLP of size $n+m$. On the assumption that $n \geq m$, it takes $O\left(n^{4} \log n\right)$ time and $O\left(n^{3}\right)$ space in total.

\subsection{The $F M$ function}

For any two variables $X_{i}, Y_{j}$ and integer $k$ with $1 \leq k \leq\left|X_{i}\right|$, we define the function $F M\left(X_{i}, Y_{j}, k\right)$ which returns the previous position of the first position of mismatches, when we compare $Y_{j}$ with $X_{i}$ at position $k$. Formally,

$$
F M\left(X_{i}, Y_{j}, k\right)=\min \left\{1 \leq h \leq\left|Y_{j}\right| \mid X_{i}[k+h-1] \neq Y_{j}[h]\right\}-1 .
$$

Namely, $F M\left(X_{i}, Y_{j}, k\right)$ equals the length of the common prefix of $X_{i}\left[k:\left|X_{i}\right|\right]$ and $Y_{j}$ when it is not zero. When the common prefix is the empty string $\varepsilon$ (when such $h$ does not exist), let $F M\left(X_{i}, Y_{j}, k\right)=0$.

Lemma 3 ([9]). For any variables $X_{i}, Y_{j}$ and integer $k, F M\left(X_{i}, Y_{j}, k\right)$ can be computed in $O(n \log n)$ time, provided that $O L\left(X_{i^{\prime}}, X_{j^{\prime}}\right)$ is already computed for any $1 \leq i^{\prime} \leq i$ and $1 \leq j^{\prime} \leq j$. 

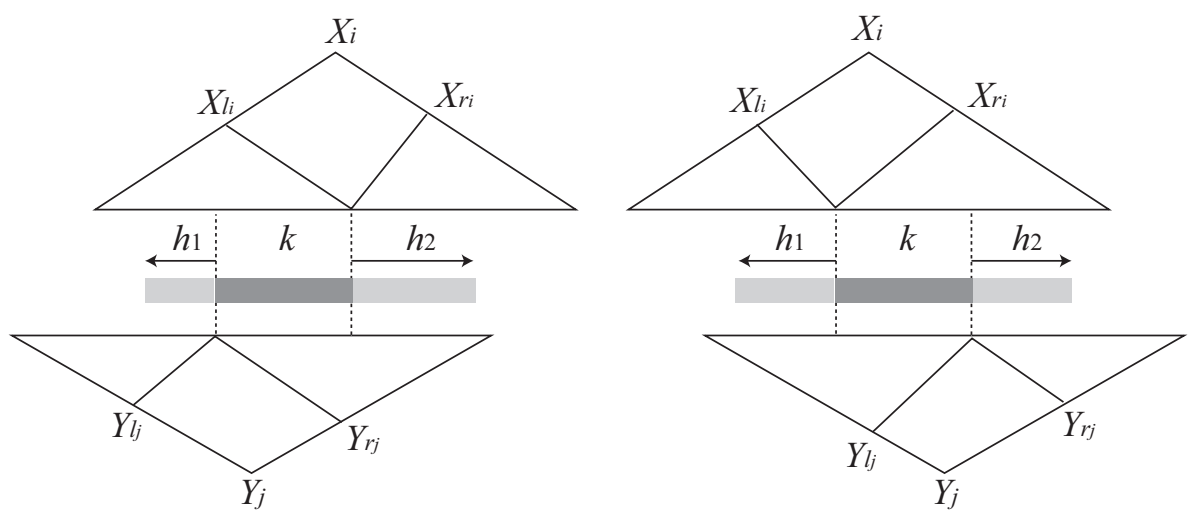

Fig. 1. Illustration of Observation 2 where we "extend" an overlap $k$ as a candidate of $\operatorname{LCStr}(T, S)$.

\subsection{Efficient Computation of Longest Common Substrings}

The main idea of our algorithm for computing $\operatorname{LCStr}(T, S)$ is based on the following observation.

Observation 1 For any substring $Z$ of string $T$, there always exists a variable $X_{i}=X_{\ell_{i}} X_{r_{i}}$ of $S L P \mathcal{T}$ such that:

$-Z$ is a substring of $X_{i}$ and

- $Z$ touches or covers the boundary between $X_{\ell_{i}}$ and $X_{r_{i}}$.

It directly follows from the above observation that any common substring of strings $T, S$ touches or covers both of the boundaries in $X_{i}$ and $Y_{j}$ for some $1 \leq i \leq n$ and $1 \leq j \leq m$.

For any SLP variables $X_{i}=X_{\ell_{i}} X_{r_{i}}$ and $Y_{j}=Y_{\ell_{j}} Y_{r_{j}}$, and $k \in O L\left(X_{i}, Y_{j}\right)$, let $\operatorname{Ext}_{X_{i}, Y_{j}}(k)=k+h_{1}+h_{2}$ such that $h_{1}=\operatorname{LCSuf}\left(X_{\ell_{i}}\left[1:\left|X_{\ell_{i}}\right|-k\right], Y_{\ell_{j}}\right)$ and $h_{2}=$ $L C P r e f\left(X_{r_{i}}, Y_{r_{j}}\left[k+1:\left|Y_{r_{j}}\right|\right]\right)$. For any $k \notin O L\left(X_{i}, Y_{j}\right)$, we leave $\operatorname{Ext}_{X_{i}, Y_{j}}(k)$ undefined. For a set $S$ of integers, we define $\operatorname{Ext}_{X_{i}, Y_{j}}(S)=\left\{\operatorname{Ext}_{X_{i}, Y_{j}}(k) \mid k \in S\right\}$. $\operatorname{Ext}_{Y_{j}, X_{i}}(k)$ and $\operatorname{Ext}_{Y_{j}, X_{i}}(S)$ are defined similarly.

The next observation follows from the above arguments (see also Fig. 1):

Observation 2 For any strings $T$ and $S, L C S t r(T, S)$ equals to the maximum element of the set

$$
\begin{gathered}
\bigcup_{1 \leq i \leq n, 1 \leq j \leq m}\left(\operatorname{Ext}_{X_{i}, Y_{j}}\left(O L\left(X_{\ell_{i}}, Y_{r_{j}}\right)\right) \cup \operatorname{Ext}_{Y_{j}, X_{i}}\left(O L\left(Y_{\ell_{j}}, X_{r_{i}}\right)\right) \cup \operatorname{LCStr}^{*}\left(X_{i}, Y_{j}\right)\right), \\
\text { where } \operatorname{LCStr}{ }^{*}\left(X_{i}, Y_{j}\right)=\operatorname{LCSuf}\left(X_{\ell_{i}}, Y_{\ell_{j}}\right)+\operatorname{LCPref}\left(X_{r_{i}}, Y_{r_{j}}\right) .
\end{gathered}
$$

Based on Observation 2, our strategy for computing $\operatorname{LCStr}(T, S)$ is to compute $\max \left(E x t_{X_{i}, Y_{j}}\left(O L\left(X_{\ell_{i}}, Y_{r_{j}}\right)\right)\right)$ and $\max \left(\operatorname{Ext}_{Y_{j}, X_{i}}\left(O L\left(Y_{\ell_{j}}, X_{r_{i}}\right)\right)\right)$ for each pair of $X_{i}$ and $Y_{j}$. Lemma 4 shows how to compute $\max \left(\operatorname{Ext}_{X_{i}, Y_{j}}\left(O L\left(X_{\ell_{i}}, Y_{r_{j}}\right)\right)\right)$ and $\max \left(\operatorname{Ext}_{Y_{j}, X_{i}}\left(O L\left(Y_{\ell_{j}}, X_{r_{i}}\right)\right)\right)$ using $F M$. 


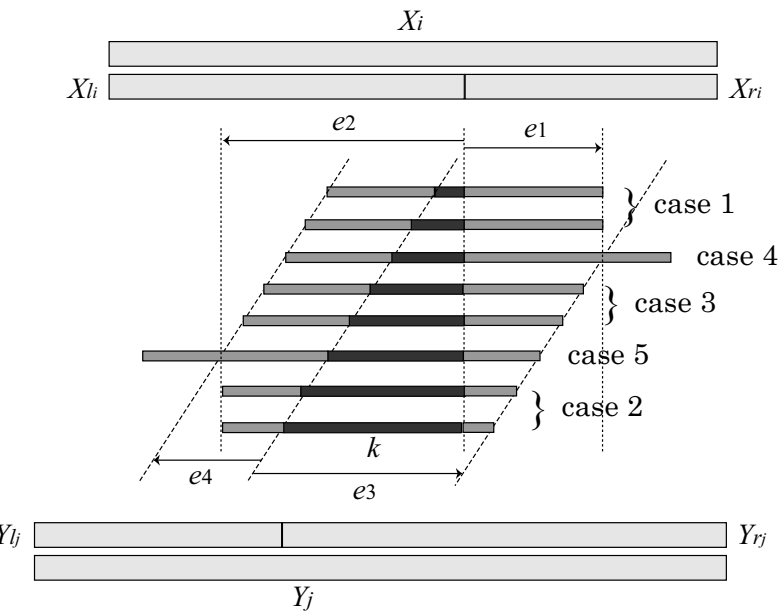

Fig. 2. Illustration for the proof of Lemma 4. The dark rectangles represent the overlaps between $X_{\ell_{i}}$ and $Y_{r_{j}}$. Case 6 is the special case where cases 4 and 5 happen at the same time and case 3 does not exist.

Lemma 4. For any variables $X_{i}=X_{\ell_{i}} X_{r_{i}}$ and $Y_{j}=Y_{\ell_{j}} Y_{r_{j}}$, we can compute $\max \left(\operatorname{Ext}_{X_{i}, Y_{j}}\left(O L\left(X_{\ell_{i}}, Y_{r_{j}}\right)\right)\right)$ and $\max \left(\operatorname{Ext}_{Y_{j}, X_{i}}\left(O L\left(Y_{\ell_{j}}, X_{r_{i}}\right)\right)\right)$ in $O\left(n^{2} \log n\right)$ time.

Proof. Here we concentrate on computing $\max \left(\operatorname{Ext}_{X_{i}, Y_{j}}\left(O L\left(X_{\ell_{i}}, Y_{r_{j}}\right)\right)\right)$, as the case of $\max \left(\operatorname{Ext}_{Y_{j}, X_{i}}\left(O L\left(Y_{\ell_{j}}, X_{r_{i}}\right)\right)\right)$ is just symmetric. Let $\langle a, d, t\rangle$ be any of the $O(n)$ arithmetic progressions of $O L\left(X_{\ell_{i}}, Y_{r_{j}}\right)$.

Assume that $t>1$ and $a<d$. The cases where $t=1$ or $a=d$ are easier to show. Let $u=Y_{r_{j}}[1: a]$ and $v=Y_{r_{j}}[a+1: d]$. For any string $w$, let $w^{*}$ denote an infinite repetition of $w$, that is, $w^{*}=w w w \cdots$. Firstly we compute

$e_{1}=L \operatorname{CPref}\left(X_{r_{i}},(v u)^{*}\right)= \begin{cases}F M\left(Y_{r_{j}}, X_{r_{i}}, a+1\right) & \text { if } F M\left(Y_{r_{j}}, X_{r_{i}}, a+1\right)<d, \\ F M\left(X_{r_{i}}, X_{r_{i}}, d+1\right)+d & \text { otherwise, }\end{cases}$

$e_{2}=\operatorname{LCSuf}\left(X_{\ell_{i}},(v u)^{*}\right)=F M\left(X_{\ell_{i}}^{R}, X_{\ell_{i}}^{R}, d+1\right)+d$,

$e_{3}=\operatorname{LCPref}\left(Y_{r_{j}},(u v)^{*}\right)=F M\left(Y_{r_{j}}, Y_{r_{j}}, d+1\right)+d$,

$e_{4}=\operatorname{LCSuf}\left(Y_{\ell_{j}},(u v)^{*}\right)= \begin{cases}F M\left(X_{\ell_{i}}^{R}, Y_{\ell_{j}}^{R}, a+1\right) & \text { if } F M\left(X_{\ell_{i}}^{R}, Y_{\ell_{j}}^{R}, a+1\right)<d, \\ F M\left(Y_{\ell_{j}}^{R}, Y_{\ell_{j}}^{R}, d+1\right)+d & \text { otherwise. }\end{cases}$

(See also Fig. 2.) As above, we can compute $e_{1}, e_{2}, e_{3}, e_{4}$ by at most 6 calls of $F M$. Note that $X_{i}\left[\left|X_{\ell_{i}}\right|-e_{2}+1:\left|X_{\ell_{i}}\right|+e_{1}\right]$ is the longest substring of $X_{i}$ that contains $X_{i}\left[\left|X_{\ell_{i}}\right|-d+1:\left|X_{\ell_{i}}\right|\right]$ and has a period $d$. Note also that $Y_{j}\left[\left|Y_{\ell_{j}}\right|-e_{4}+1\right.$ : $\left.\left|Y_{\ell_{j}}\right|+e_{3}\right]$ is the longest substring of $Y_{j}$ that contains $Y_{j}\left[\left|Y_{\ell_{j}}\right|+1:\left|Y_{\ell_{j}}\right|+d\right]$ and has a period $d$.

Let $k \in\langle a, d, t\rangle$. We categorize $\operatorname{Ext}_{X_{i}, Y_{j}}(k)$ depending on the value of $k$, as follows. 
case 1: When $k<\min \left\{e_{3}-e_{1}, e_{2}-e_{4}\right\}$. If $k-d \in\langle a, d, t\rangle$, it is not difficult to see $\operatorname{Ext}_{X_{i}, Y_{j}}(k)=\operatorname{Ext}_{X_{i}, Y_{j}}(k-d)+d$. Therefore, we have

$$
A=\max \left\{\operatorname{Ext}_{X_{i}, Y_{j}}(k) \mid k<\min \left\{e_{3}-e_{1}, e_{2}-e_{4}\right\}\right\}=\operatorname{Ext}_{X_{i}, Y_{j}}\left(k^{\prime}\right),
$$

where $k^{\prime}=\max \left\{k \mid k<\min \left\{e_{3}-e_{1}, e_{2}-e_{4}\right\}\right\}$.

case 2: When $k>\max \left\{e_{3}-e_{1}, e_{2}-e_{4}\right\}$. If $k+d \in\langle a, d, t\rangle$, it is not difficult to see $\operatorname{Ext}_{X_{i}, Y_{j}}(k)=\operatorname{Ext}_{X_{i}, Y_{j}}(k+d)+d$. Therefore, we have

$$
B=\max \left\{\operatorname{Ext}_{X_{i}, Y_{j}}(k) \mid k>\max \left\{e_{3}-e_{1}, e_{2}-e_{4}\right\}\right\}=\operatorname{Ext}_{X_{i}, Y_{j}}\left(k^{\prime \prime}\right),
$$

where $k^{\prime \prime}=\min \left\{k \mid k>\max \left\{e_{3}-e_{1}, e_{2}-e_{4}\right\}\right\}$.

case 3: When $\min \left\{e_{3}-e_{1}, e_{2}-e_{4}\right\}<k<\max \left\{e_{3}-e_{1}, e_{2}-e_{4}\right\}$. In this case we have $\operatorname{Ext}_{X_{i}, Y_{j}}(k)=\min \left\{e_{1}+e_{2}, e_{3}+e_{4}\right\}$ for any $k$ with $\min \left\{e_{3}-e_{1}, e_{2}-e_{4}\right\}<$ $k<\max \left\{e_{3}-e_{1}, e_{2}-e_{4}\right\}$. Thus

$$
\begin{aligned}
C & =\max \left\{\operatorname{Ext}_{X_{i}, Y_{j}}(k) \mid \min \left\{e_{3}-e_{1}, e_{2}-e_{4}\right\}<k<\max \left\{e_{3}-e_{1}, e_{2}-e_{4}\right\}\right\} \\
& =\min \left\{e_{1}+e_{2}, e_{3}+e_{4}\right\} .
\end{aligned}
$$

case 4: When $k=e_{3}-e_{1}$. In this case we have

$$
\begin{aligned}
D=\operatorname{Ext}_{X_{i}, Y_{j}}(k) & =k+\min \left\{e_{2}-k, e_{4}\right\}+\operatorname{LCPref}\left(Y_{r_{j}}\left[k+1:\left|Y_{r_{j}}\right|\right], X_{r_{i}}\right) \\
& =k+\min \left\{e_{2}-k, e_{4}\right\}+\operatorname{FM}\left(Y_{r_{j}}, X_{r_{i}}, k+1\right) .
\end{aligned}
$$

case 5: When $k=e_{2}-e_{4}$. In this case we have

$$
\begin{aligned}
E=\operatorname{Ext}_{X_{i}, Y_{j}}(k) & =k+\operatorname{LCSuf}\left(X_{\ell_{i}}\left[1:\left|X_{\ell_{i}}\right|-k\right], Y_{\ell_{j}}\right)+\min \left\{e_{1}, e_{3}-k\right\} \\
& =k+\operatorname{FM}\left(X_{\ell_{i}}^{R}, Y_{\ell_{j}}^{R}, k+1\right)+\min \left\{e_{1}, e_{3}-k\right\} .
\end{aligned}
$$

case 6: When $k=e_{3}-e_{1}=e_{2}-e_{4}$. In this case we have

$$
\begin{aligned}
F & =\operatorname{Ext}_{X_{i}, Y_{j}}(k) \\
& =k+\operatorname{LCSuf}\left(X_{\ell_{i}}\left[1:\left|X_{\ell_{i}}\right|-k\right], Y_{\ell_{j}}\right)+\operatorname{LCPref}\left(Y_{r_{j}}\left[k+1:\left|Y_{r_{j}}\right|\right], X_{r_{i}}\right) \\
& =k+F M\left(X_{\ell_{i}}^{R}, Y_{\ell_{j}}^{R}, k+1\right)+F M\left(Y_{r_{j}}, X_{r_{i}}, k+1\right) .
\end{aligned}
$$

Then clearly the following inequality stands (see also Fig. 2):

$$
F \geq \max \{D, E\} \geq C \geq \max \{A, B\} .
$$

A membership query to the arithmetic progression $\langle a, d, t\rangle$ can be answered in constant time. Also, an element $k \in\langle a, d, t\rangle$ such that $\min \left\{e_{3}-e_{1}, e_{2}-e_{4}\right\}<$ $k<\max \left\{e_{3}-e_{1}, e_{2}-e_{4}\right\}$ of case 3 can be found in constant time, if such exists. $k^{\prime}$ and $k^{\prime \prime}$ of case 1 and case 2, respectively, can be computed in constant time as well. Therefore, based on inequality (1), we can compute $\max \left(\operatorname{Ext}_{X_{i}, Y_{j}}(\langle a, d, t\rangle)\right)$ by at most 2 calls of $F M$, provided that $e_{1}, e_{2}, e_{3}, e_{4}$ are already computed.

Since $O L\left(X_{\ell_{i}}, Y_{r_{j}}\right)$ contains $O(n)$ arithmetic progressions by Lemma 2, and each call of $F M$ takes $O(n \log n)$ time by Lemma $3, \max \left(E_{X_{X_{i}, Y_{j}}}\left(O L\left(X_{\ell_{i}}, Y_{r_{j}}\right)\right)\right)$ can be computed in $O\left(n^{2} \log n\right)$ time. 
Now we obtain the main result of this section.

Theorem 2. Problem 1 can be solved in $O\left(n^{4} \log n\right)$ time with $O\left(n^{3}\right)$ space.

Proof. It follows from Theorem 1 that $O L\left(X_{i}, Y_{j}\right)$ can be computed in $O\left(n^{4} \log n\right)$ time with $O\left(n^{3}\right)$ space. For any variables $X_{i}=X_{\ell_{i}} X_{r_{i}}$ and $Y_{j}=Y_{\ell_{j}} Y_{r_{j}}$, by Lemmas 2, 3 and 4, $\max \left(\operatorname{Ext}_{X_{i}, Y_{j}}\left(O L\left(X_{\ell_{i}}, Y_{r_{j}}\right)\right)\right)$ and $\max \left(\operatorname{Ext}_{Y_{j}, X_{i}}\left(O L\left(Y_{\ell_{j}}, X_{r_{i}}\right)\right)\right)$ can be computed in $O\left(n^{2} \log n\right)$ time.

Moreover, it is easy to see that

$$
\begin{aligned}
\operatorname{LCSuf}\left(X_{\ell_{i}}, Y_{\ell_{j}}\right) & =F M\left(X_{\ell_{i}}^{R}, Y_{\ell_{j}}^{R}, 1\right) \text { and } \\
\operatorname{LCPref}\left(X_{r_{i}}, Y_{r_{j}}\right) & =F M\left(X_{r_{i}}, Y_{r_{j}}, 1\right) .
\end{aligned}
$$

Thus $\operatorname{LCStr}^{*}\left(X_{i}, Y_{j}\right)$ can be computed in $O(n \log n)$ time. Overall, by Observation 2 it takes $O\left(n^{4} \log n\right)$ time and $O\left(n^{3}\right)$ to solve Problem 1.

The following corollary is immediate.

Corollary 1. Given two $S L P s \mathcal{T}$ and $\mathcal{S}$ describing strings $T$ and $S$ respectively, the beginning and ending positions of a longest common substring of $T$ and $S$ can be computed in $O\left(n^{4} \log n\right)$ time with $O\left(n^{3}\right)$ space.

\section{Computing Palindromes from SLP Compressed Strings}

In this section we present an efficient algorithm that computes a succinct representation of all maximal palindromes of string $T$, when its corresponding SLP $\mathcal{T}$ is given as input. The algorithm runs in $O\left(n^{4}\right)$ time and $O\left(n^{2}\right)$ space, where $n$ is the size of the input SLP $\mathcal{T}$.

For any string $T$, let Pals $(T)$ denote the set of pairs of the beginning and ending positions of all maximal palindromes in $T$, namely,

$\operatorname{Pals}(T)=\left\{(p, q) \mid T[p: q]\right.$ is the maximal palindrome centered at $\left.\left\lfloor\frac{p+q}{2}\right\rfloor\right\}$.

Note that the size of $\operatorname{Pals}(T)$ is $O(|T|)=O\left(2^{n}\right)$. Thus we introduce a succinct representation of $\operatorname{Pals}(T)$ in the next subsection.

\subsection{Succinct Representation of Pals $(T)$}

Let $X_{i}$ denote a variable in $\mathcal{T}$ for $1 \leq i \leq n$. For any variables $X_{i}=X_{\ell} X_{r}$, let $\mathrm{Pals}^{\triangle}\left(X_{i}\right)$ be the set of pairs of beginning and ending positions of maximal palindromes of $X_{i}$ that cover or touch the boundary between $X_{\ell}$ and $X_{r}$, namely,

$$
\text { Pals }^{\triangle}\left(X_{i}\right)=\left\{(p, q) \in \operatorname{Pals}\left(X_{i}\right)|1 \leq p \leq| X_{\ell}|+1,| X_{\ell}|\leq q \leq| X_{i} \mid, p \leq q\right\} .
$$

Also, let PPals $(T)$ and SPals $(T)$ denote the set of pairs of the beginning and ending positions of the prefix and suffix palindromes of $T$, respectively, that is,

$$
\begin{aligned}
& \operatorname{PPals}(T)=\{(1, q) \in \operatorname{Pals}(T)|1 \leq q \leq| T \mid\}, \text { and } \\
& \operatorname{SPals}(T)=\{(p,|T|) \in \operatorname{Pals}(T)|1 \leq p \leq| T \mid\} .
\end{aligned}
$$

Ga̧sieniec et al. [5] claimed the following lemma: 
Lemma 5 ([5]). For any string $T, P P a l s(T)$ and $S P a l s(T)$ can be represented by $O(\log |T|)$ arithmetic progressions.

We have the following observation for decomposition of $\operatorname{Pals}\left(X_{i}\right)$.

Observation 3 For any variables $X_{i}=X_{\ell} X_{r}$,

$$
\begin{aligned}
\operatorname{Pals}\left(X_{i}\right)= & \left(\operatorname{Pals}\left(X_{\ell}\right)-\operatorname{SPals}\left(X_{\ell}\right)\right) \cup \\
& \operatorname{Pals}^{\triangle}\left(X_{i}\right) \cup\left(\left(\operatorname{Pals}\left(X_{r}\right)-\operatorname{PPals}\left(X_{r}\right)\right) \oplus\left|X_{\ell}\right|\right) .
\end{aligned}
$$

Thus, the desired output Pals $(T)=\operatorname{Pals}\left(X_{n}\right)$ can be represented as a combination of $\left\{\text { Pals }^{\triangle}\left(X_{i}\right)\right\}_{i=1}^{n},\left\{\operatorname{PPals}\left(X_{i}\right)\right\}_{i=1}^{n}$ and $\left\{\operatorname{SPals}\left(X_{i}\right)\right\}_{i=1}^{n}$. Therefore, computing Pals $(T)$ is reduced to computing Pals $\triangle\left(X_{i}\right), \operatorname{PPals}\left(X_{i}\right)$ and $\operatorname{SPals}\left(X_{i}\right)$, for every $i=1,2, \ldots, n$. The problem to be tackled in this section follows:

Problem 2. Given an SLP $\mathcal{T}$ of size $n$, compute $\left\{\text { Pals }^{\triangle}\left(X_{i}\right)\right\}_{i=1}^{n},\left\{\operatorname{PPals}\left(X_{i}\right)\right\}_{i=1}^{n}$ and $\left\{\operatorname{SPals}\left(X_{i}\right)\right\}_{i=1}^{n}$.

Lemma 6 is useful to compute $\operatorname{Pals}^{\triangle}\left(X_{i}\right)$ from $\operatorname{SPals}\left(X_{\ell}\right)$ and $\operatorname{PPals}\left(X_{r}\right)$.

Lemma 6. For any variable $X_{i}=X_{\ell} X_{r}$ and any $(p, q) \in$ Pals $^{\triangle}\left(X_{i}\right)$, there exists an integer $l \geq 0$ such that $(p+l, q-l) \in \operatorname{SPals}\left(X_{\ell}\right) \cup\left(\operatorname{PPals}\left(X_{r}\right) \oplus\left|X_{\ell}\right|\right) \cup$ $\left\{\left(\left|X_{\ell}\right|,\left|X_{\ell}\right|+1\right)\right\}$.

Proof. Since $X_{i}[p: q]$ is a palindrome, $X_{i}[p+l: q-l]$ is also a palindrome for any $0 \leq l<\left\lfloor\frac{p+q}{2}\right\rfloor$. Then we have the following three cases:

1. When $\left\lfloor\frac{p+q}{2}\right\rfloor<\left|X_{\ell}\right|$, for $l=p-\left|X_{\ell}\right|$, we have $(p+l, q-l) \in \operatorname{SPals}\left(X_{\ell}\right)$.

2. When $\left\lfloor\frac{p+q}{2}\right\rfloor>\left|X_{\ell}\right|$, for $l=\left|X_{\ell}\right|-p+1$, we have $(p+l, q-l) \in \operatorname{PPals}\left(X_{r}\right)$.

3. When $\left\lfloor\frac{p+q}{2}\right\rfloor=\left|X_{\ell}\right|$, if $q-p+1$ is odd, then the same arguments to case 1 apply, since $X_{\ell}\left[\left|X_{\ell}\right|\right]=X_{\ell}\left[\left|X_{\ell}\right|\right]^{R}$ and $\left(\left|X_{\ell}\right|,\left|X_{\ell}\right|\right) \in \operatorname{SPals}\left(X_{\ell}\right)$. If $q-p+1$ is even, let $l=\left|X_{\ell}\right|-p$. In this case, we have $p+q=2\left|X_{\ell}\right|+1$. Thus, $p+l=\left|X_{\ell}\right|$ and $q-l=\left|X_{\ell}\right|+1$.

By Lemma 6, Pals $^{\triangle}\left(X_{i}\right)$ can be computed by "extending" all palindromes in SPals $\left(X_{\ell}\right)$ and PPals $\left(X_{r}\right)$ to the maximal within $X_{i}$, and finding the maximal even palindromes centered at $\left|X_{\ell}\right|$ in $X_{i}$. In so doing, for any (maximal or non-maximal) palindrome $P=X_{i}[p: q]$, we define function $\operatorname{Ext}_{X_{i}}$ so that $\operatorname{Ext}_{X_{i}}(p, q)=(p-h, q+h)$, where $h \geq 0$ and $X_{i}[p-h: q+h]$ is the maximal palindrome centered at position $\left\lfloor\frac{p+q}{2}\right\rfloor$ in $X_{i}$. For any $p, q$ with $X_{i}[p: q]$ not being a palindrome, we leave $\operatorname{Ext}_{X_{i}}(p, q)$ undefined. For a set $S$ of pair of integers, let $\operatorname{Ext}_{X_{i}}(S)=\left\{\operatorname{Ext}_{X_{i}}(p, q) \mid(p, q) \in S\right\}$.

The next observations give us a recursive procedure to compute $\mathrm{Pals}^{\triangle}\left(X_{i}\right)$.

Observation 4 For any variable $X_{i}=X_{\ell} X_{r}$,

$$
\begin{gathered}
\text { Pals }^{\triangle}\left(X_{i}\right)=\operatorname{Ext}_{X_{i}}\left(\operatorname{SPals}\left(X_{\ell}\right)\right) \cup \operatorname{Ext}_{X_{i}}\left(\operatorname{PPals}\left(X_{r}\right)\right) \cup \text { Pals }^{*}\left(X_{i}\right), \text { where } \\
\text { Pals }^{*}\left(X_{i}\right)=\left\{\left(\left|X_{\ell}\right|-l+1,\left|X_{\ell}\right|+l\right) \in \operatorname{Pals}\left(X_{i}\right) \mid l \geq 1\right\} .
\end{gathered}
$$


$\operatorname{PPals}\left(X_{i}\right)$ and $\operatorname{SPals}\left(X_{i}\right)$ can be computed from $\operatorname{Pals}^{\triangle}\left(X_{i}\right)$ as follows:

Observation 5 For any variable $X_{i}=X_{\ell} X_{r}$,

$$
\begin{aligned}
& \operatorname{PPals}\left(X_{i}\right)=\operatorname{PPals}\left(X_{\ell}\right) \cup\left\{(1, q) \in \text { Pals }^{\triangle}\left(X_{i}\right)\right\} \text { and } \\
& \operatorname{SPals}\left(X_{i}\right)=\left(\operatorname{SPals}\left(X_{r}\right) \oplus\left|X_{\ell}\right|\right) \cup\left\{\left(p,\left|X_{i}\right|\right) \in \text { Pals }^{\triangle}\left(X_{i}\right)\right\} .
\end{aligned}
$$

\subsection{Efficient Computation of $\mathrm{Pals}^{\triangle}\left(\mathrm{X}_{i}\right)$}

Let us first briefly recall the work of $[10,11]$. For any variables $X_{i}=X_{\ell} X_{r}$ and $X_{j}$, we define the set $O c c^{\triangle}\left(X_{i}, X_{j}\right)$ of all occurrences of $X_{j}$ that cover or touch the boundary between $X_{\ell}$ and $X_{r}$, namely,

$$
O c c^{\triangle}\left(X_{i}, X_{j}\right)=\left\{s>0\left|X_{i}\left[s: s+\left|X_{j}\right|-1\right]=X_{j},\right| X_{\ell}|-| X_{j}|+1 \leq s \leq| X_{\ell} \mid\right\} .
$$

Theorem 3 ([11]). For any variables $X_{i}$ and $X_{j}, O c c^{\triangle}\left(X_{i}, X_{j}\right)$ can be computed in total of $O\left(n^{3}\right)$ time and $O\left(n^{2}\right)$ space.

Lemma 7 ([10]). For any variables $X_{i}, X_{j}$ and integer $k, F M\left(X_{i}, X_{j}, k\right)$ can be computed in $O\left(n^{2}\right)$ time, provided that $\operatorname{Occ}^{\triangle}\left(X_{i^{\prime}}, X_{j^{\prime}}\right)$ is already computed for any $1 \leq i^{\prime} \leq i$ and $1 \leq j^{\prime} \leq j$.

Lemma 8. For any variable $X_{i}=X_{\ell} X_{r}$ and any arithmetic progression $\langle a, d, t\rangle$ with $(1,\langle a, d, t\rangle) \subseteq P$ Pals $\left(X_{r}\right)$, Ext $_{X_{i}}((1,\langle a, d, t\rangle))$ can be represented by at most 2 arithmetic progressions and a pair of the beginning and ending positions of a maximal palindrome, and can be computed by at most 4 calls of FM. Similar for $\operatorname{Ext}_{X_{i}}\left(\left(\langle a, d, t\rangle,\left|X_{\ell}\right|\right)\right)$ with $\left(\left\langle a^{\prime}, d^{\prime}, t^{\prime}\right\rangle,\left|X_{\ell}\right|\right) \subseteq \operatorname{SPals}\left(X_{\ell}\right)$.

Proof. By Lemma 3.4 of [13].

We are now ready to prove the following lemma:

Lemma 9. For any variable $X_{i}=X_{\ell} X_{r}$, Pals $^{\triangle}\left(X_{i}\right)$ requires $O\left(\log \left|X_{i}\right|\right)$ space and can be computed in $O\left(n^{2} \log \left|X_{i}\right|\right)$ time.

Proof. Recall Observation 4. It is clear from the definition that $\operatorname{Pals}^{*}\left(X_{i}\right)$ is either singleton or empty. When it is a singleton, it consists of the maximal even palindrome centered at $\left|X_{\ell}\right|$. Let $l=F M\left(X_{r}, X_{\ell}^{R}, 1\right)$. Then we have

$$
\text { Pals }^{*}\left(X_{i}\right)= \begin{cases}\emptyset & \text { if } l=0, \\ \left\{\left(\left|X_{\ell}\right|-l+1,\left|X_{\ell}\right|+l\right)\right\} & \text { otherwise. }\end{cases}
$$

Due to Lemma 7, Pals $^{*}\left(X_{i}\right)$ can be computed in $O\left(n^{2}\right)$ time.

Now we consider $\operatorname{Ext}_{X_{i}}\left(\operatorname{SPals}\left(X_{\ell}\right)\right)$. By Lemma 7 and Lemma 8, each subset $\operatorname{Ext}_{X_{i}}((1,\langle a, d, t\rangle)) \subseteq \operatorname{Ext}_{X_{i}}\left(S P a l s\left(X_{\ell}\right)\right)$ requires $O(1)$ space and can be computed in $O\left(n^{2}\right)$ time. It follows from Lemma 5 that $\operatorname{Ext}_{X_{i}}\left(\operatorname{SPals}\left(X_{\ell}\right)\right)$ consists of $O\left(\log \left|X_{i}\right|\right)$ arithmetic progressions. Thus $\operatorname{Ext}_{X_{i}}\left(\operatorname{SPals}\left(X_{\ell}\right)\right)$ can be computed in $O\left(n^{2} \log \left|X_{i}\right|\right)$ time. Similar arguments hold for $\operatorname{Ext}_{X_{i}}\left(\operatorname{PPals}\left(X_{r}\right)\right)$. 


\subsection{Results}

Theorem 4. Problem 2 can be solved in $O\left(n^{4}\right)$ time with $O\left(n^{2}\right)$ space.

Proof. Firstly we analyze the time complexity. From Theorem 3 preprocessing for the $F M$ function takes $O\left(n^{3}\right)$ time. By Lemma 7, each call of $F M$ takes $O\left(n^{2}\right)$ time. It follows from Lemma 9 that $\mathrm{Pals}^{\triangle}\left(X_{i}\right)$ can be computed in $O\left(n^{3}\right)$ time. By Observation 5, PPals $\left(X_{i}\right)$ and $\operatorname{SPals}\left(X_{i}\right)$ can be computed in $O(n)$ time from Pals $^{\triangle}\left(X_{i}\right)$. Hence the overall time cost to compute $\left\{P \operatorname{Pals}\left(X_{i}\right)\right\}_{i=1}^{n}$, $\left\{\operatorname{SPals}\left(X_{i}\right)\right\}_{i=1}^{n}$, and $\left\{\right.$ Pals $\left.^{\triangle}\left(X_{i}\right)_{i=1}^{n}\right\}$ is $O\left(n^{4}\right)$.

Secondly we analyze the space complexity. The preprocessing for the $F M$ function requires $O\left(n^{2}\right)$ due to Theorem 3. From Lemma 5 PPals $\left(X_{i}\right)$ and $\operatorname{SPals}\left(X_{i}\right)$ require $O(n)$ space. Lemma 9 states that $\mathrm{Pals}^{\triangle}\left(X_{i}\right)$ requires $O(n)$ space. Thus the total space requirement is $O\left(n^{2}\right)$.

The following two theorems are results obtained by slightly modifying the algorithm of the previous subsections.

Theorem 5. Given an SLP $\mathcal{T}$ that describes string $T$, whether $T$ is a palindrome or not can be determined with extra $O(1)$ space and without increasing asymptotic time complexities of the algorithm.

Proof. It suffices to see if $(1,|T|) \in \operatorname{PPals}(T)=\operatorname{PPals}\left(X_{n}\right)$. By Lemma 5, PPals $\left(X_{n}\right)$ can be represented by $O(n)$ arithmetic progressions. It is not difficult to see that $T$ is a palindrome if and only if $a+(t-1) d=|T|$ for the arithmetic progression $\langle a, d, t\rangle$ of the largest common difference among those in PPals $\left(X_{n}\right)$. Such an arithmetic progression can easily be found during computation of PPals $\left(X_{n}\right)$ without increasing asymptotic time complexities of the algorithm.

Theorem 6. Given an $S L P \mathcal{T}$ that describes string $T$, the position pair $(p, q)$ of the longest palindrome in $T$ can be found with extra $O(1)$ space and without increasing asymptotic time complexities of the algorithm.

Proof. We compute the beginning and ending positions of the longest palindrome in $P_{a l s} \triangle\left(X_{i}\right)$ for $i=1,2, \ldots, n$. It takes $O(n)$ time for each $X_{i}$. If its length exceeds the length of the currently kept palindrome, we update the beginning and ending positions.

Provided that $\left\{\operatorname{PPals}\left(X_{i}\right)\right\}_{i=1}^{n},\left\{\operatorname{SPals}\left(X_{i}\right)\right\}_{i=1}^{n}$, and $\left\{\operatorname{Pals}^{\triangle}\left(X_{i}\right)_{i=1}^{n}\right\}$ are already computed, we have the following result:

Theorem 7. Given pair $(p, q)$ of integers, it can be answered in $O(n)$ time whether or not substring $T[p: q]$ is a maximal palindrome of $T$.

Proof. We binary search the derivation tree of SLP $\mathcal{T}$ until finding the variable $X_{i}=X_{\ell} X_{r}$ such that $1+$ offset $\leq p \leq\left|X_{\ell}\right|+$ offset and $1+$ offset $+\left|X_{\ell}\right| \leq q \leq$ $\left|X_{i}\right|+$ offset. This takes $O(n)$ time. Due to Observation 4, for each variable $X_{i}$, $P_{a l s} \triangle\left(X_{i}\right)$ can be represented by $O(n)$ arithmetic progressions plus a pair of the beginning and ending positions of a maximal palindrome. Thus, we can check if $(p, q) \in$ Pals $^{\triangle}\left(X_{i}\right)$ in $O(n)$ time. 
Finally we supply pseudo-codes of our algorithms.

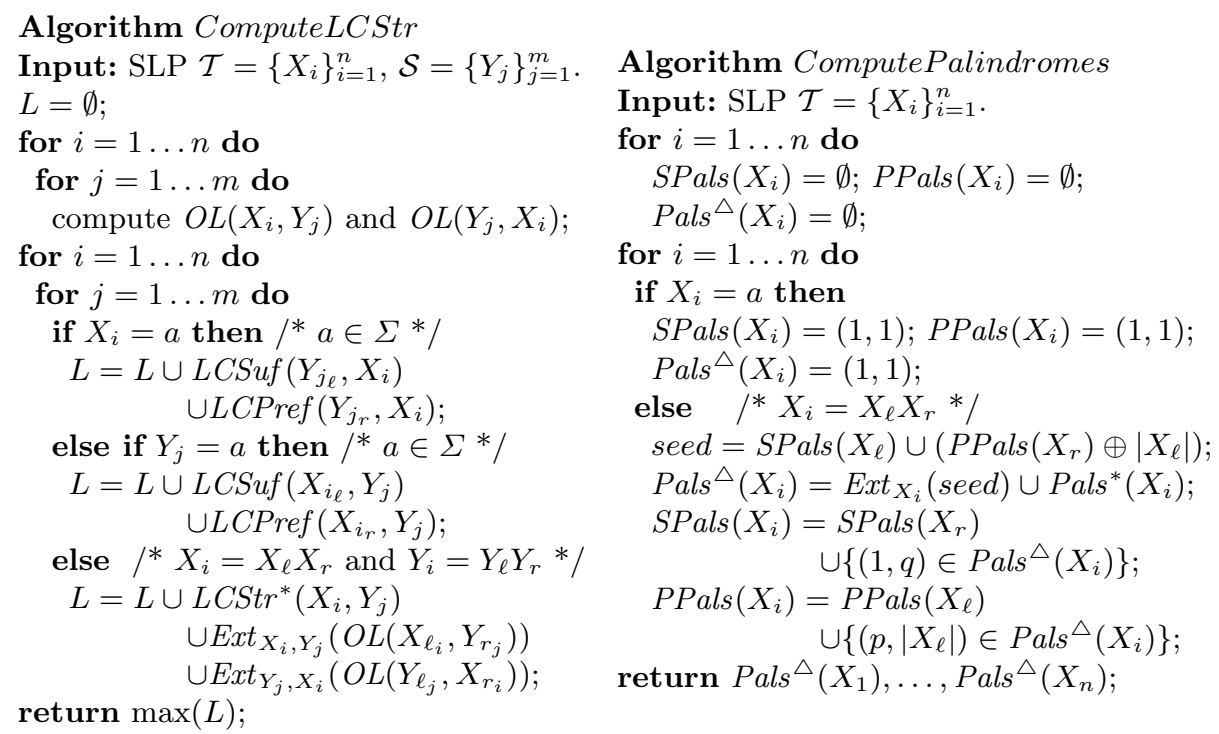

\section{References}

1. Ziv, J., Lempel, A.: A universal algorithm for sequential data compression. IEEE Trans. Info. Theory IT-23(3) (1977) 337-349

2. Ziv, J., Lempel, A.: Compression of individual sequences via variable-length coding. IEEE Trans. Info. Theory 24(5) (1978) 530-536

3. Kieffer, J., Yang, E., Nelson, G., Cosman, P.: Universal lossless compression via multilevel pattern matching. IEEE Trans. Info. Theory 46(4) (2000) 1227-1245

4. Nevill-Manning, C.G., Witten, I.H., Maulsby, D.L.: Compression by induction of hierarchical grammars. In: DCC'94, IEEE Press (1994) 244-253

5. Gasieniec, L., Karpinski, M., Plandowski, W., Rytter, W.: Efficient algorithms for Lempel-Ziv encoding. In: SWAT'96. (1996) 392-403

6. Rytter, W.: Grammar compression, lz-encodings, and string algorithms with implicit input. In: ICALP'04. Volume 3142 of LNCS., Springer-Verlag (2004) 15-27

7. Inenaga, S., Shinohara, A., Takeda, M.: An efficient pattern matching algorithm on a subclass of context free grammars. In: DLT'04. (2004) 225-236

8. Plandowski, W.: Testing equivalence of morphisms on context-free languages. In: ESA'94. Volume 855 of LNCS., Springer-Verlag (1994) 460-470

9. Karpinski, M., Rytter, W., Shinohara, A.: An efficient pattern-matching algorithm for strings with short descriptions. Nordic Journal of Computing 4 (1997) 172-186

10. Miyazaki, M., Shinohara, A., Takeda, M.: An improved pattern matching algorithm for strings in terms of straight-line programs. In: CPM'97. Volume 1264 of LNCS., Springer-Verlag (1997) 1-11

11. Lifshits, Y.: Processing compressed texts: A tractability border. In: CPM'07. Volume 4580 of LNCS., Springer-Verlag (2007) 228-240

12. Lifshits, Y., Lohrey, M.: Querying and embedding compressed texts. In: MFCS'06. Volume 4162 of LNCS., Springer-Verlag (2006) 681-692

13. Apostolico, A., Breslauer, D., Galil, Z.: Parallel detection of all palindromes in a string. Theoretical Computer Science 141 (1995) 163-173 